\title{
Comparing Collective and Personal Professional Theories of Experienced Practitioners
}

Citation for published version (APA):

van den Bogaart, A. C. M., Mazereeuw, M., Hummel, H. G. K., \& Kirschner, P. A. (2019). Comparing Collective and Personal Professional Theories of Experienced Practitioners. Scandinavian Journal of Educational

Research, 63(3), 363-377. https://doi.org/10.1080/00313831.2017.1375007

DOI:

10.1080/00313831.2017.1375007

Document status and date:

Published: 16/04/2019

Document Version:

Peer reviewed version

Please check the document version of this publication:

- A submitted manuscript is the version of the article upon submission and before peer-review. There can be important differences between the submitted version and the official published version of record. People interested in the research are advised to contact the author for the final version of the publication, or visit the DOI to the publisher's website.

- The final author version and the galley proof are versions of the publication after peer review.

- The final published version features the final layout of the paper including the volume, issue and page numbers.

Link to publication

\section{General rights}

Copyright and moral rights for the publications made accessible in the public portal are retained by the authors and/or other copyright owners and it is a condition of accessing publications that users recognise and abide by the legal requirements associated with these rights.

- Users may download and print one copy of any publication from the public portal for the purpose of private study or research.

- You may not further distribute the material or use it for any profit-making activity or commercial gain

- You may freely distribute the URL identifying the publication in the public portal.

If the publication is distributed under the terms of Article 25fa of the Dutch Copyright Act, indicated by the "Taverne" license above, please follow below link for the End User Agreement:

https://www.ou.nl/taverne-agreement

Take down policy

If you believe that this document breaches copyright please contact us at:

pure-support@ou.nl

providing details and we will investigate your claim.

Downloaded from https://research.ou.nl/ on date: 26 Apr. 2023 
Running head: COLLECTIVE AND PERSONAL PROFESSIONAL THEORIES 1

\title{
Comparing Collective and Personal Professional Theories of Experienced Practitioners
}

\author{
Antoine C. M. van den Bogaart ${ }^{1}$, Marco Mazereeuw ${ }^{1}$ \\ Hans G.K. Hummel ${ }^{2}$ \& Paul A. Kirschner ${ }^{2}$ \\ ${ }^{1}$ NHL University of Applied Science, The Netherlands \\ ${ }^{2}$ Open University of the Netherlands
}

Version 10-01-2016

Author Note

Correspondence concerning this article should be addressed to Antoine C. M. van den Bogaart, NHL University of Applied Science, Rengerslaan 10, 8917 DD Leeuwarden, The Netherlands, E-mail: a.c.m.van.den.bogaart@nhl.nl

Key words: experience, field, Collective Professional Theories, Personal Professional Theories. 


\begin{abstract}
A characteristic of becoming a professional is the internalisation of the Collective Professional Theory (CPT), the shared insights of experienced practitioners. A problem arises when different opinions stemming from practitioners' Personal Professional Theories (PPTs), obscure a CPT's content. This study argues that groups of cooperating practitioners have well-defined CPTs, which together constitute a field. The relations between CPTs, PPTs and fields are studied in case studies under accountants and teachers. In each domain a representation of a CPT was constructed and compared with PPTs. The results indicate that CPTs are more complex than PPTs and the structure of the two fields differs locally. Compared to the CPTs of teachers, differences between the CPTs of accountants are smaller. Such insights in CPTs are important for education because socialisation in a domain may be fostered if one is aware of CPTs and the surrounding field in relation to one's PPT.
\end{abstract}




\section{Introduction}

Becoming a professional can be conceived as a socialisation process into a group of established, experienced professionals (de Bruijn \& Nieuwenhuis, 1994). An important aspect of this process is the internalisation of the body of professional, theoretical and practical knowledge, convictions and values shared by the group members (Schaap, de Bruijn, van der Schaaf, \& Kirschner, 2009). In the study mentioned such bodies are called Collective Professional Theories (CPTs) and their individual, internalised counterparts Personal Professional Theories (PPTs). PPTs develop as a result of education and experience and are stored as schemes and scripts in long-term memories, where they serve as a reference base for acting and further professionalization (Schaap et al., 2009).

However, it is not always clear what the construct CPT represents. This problem becomes manifest when a group professionals is not homogeneous, i.e. when members disagreeing on certain aspects of their common profession because they belong to different schools of thought or to different cultures (De Long \& Fahey, 2000). In several domains examples can be found showing this point not to be imaginary. For instance there is debate among accountants about the tension between their role as independent controller and the non-audit services they provide (Ashbaugh, 2003). In the domain of teaching fundamental differences of opinion about the goals and content of education have always been numerous and are often reflected in political issues (Proctor, Brownlee, \& Freebody, 2015).

Apart from the conceptual problem of how a CPT should be conceived, there are two technical problems connected to gaining insight in CPTs at all. The first technical problem concerns the representation of CPTs so that they can be studied. Van den Bogaart, Bilderbeek, Schaap, Hummel and Kirschner (in press) showed that 
the main features of PPTs can be represented well by means of open, annotated concept maps. Because CPTs and PPTs have the same kind of content, it is assumed here that such maps are also suited to represent CPTs. The concepts in these maps denote the main semantic elements of professional theories. Links between concepts clarify their meaning. Annotations with concepts by means of examples clarify their meaning further. Annotations also offer the possibility to attach procedural knowledge to concepts which figure in it (van den Bogaart, Schaap, Hummel, \& Kirschner, submitted). If a concept map represents a PPT or a CPT, it is important for content validity (Anastasia \& Urbina, 1997) that implicit knowledge, which is usually hard to reveal (Wilson \& Berne, 1999), is represented in the map. In case of PPTs, implicit knowledge can be elicited through a discussion with the producer about his/her map and then be inserted in the map (van den Bogaart et al., submitted). In case of CPTs, the map construction procedure has to reckon with implicit, shared knowledge too.

The second technical problem concerns the method to compare PPTs and CPTs. A possibility is to use the four qualities Schaap, de Bruijn, van der Schaaf, Baartman and Kirschner (2011) distinguished to characterise a PPT, on CPTs as well. The quality concreteness is the ability of the theory to support professional actions; specificity is its uniqueness for a profession and complexity is the degree its content is interconnected. The fourth quality, richness, expresses the level of uniformity with which its content is distributed over six domains of professional knowledge: vocational field, organisations, professional environment, target groups, technicalinstrumental knowledge and self-knowledge and development. Van den Bogaart et al. (in press) developed a valid and reliable method to assess the four qualities by means of a concept map representation and to express them as percentages. The four qualities have a common characteristic: they are completely determined by a 
representation and not (also) by data outside of it. For this reason these qualities are called intrinsic. A CPT and a PPT can be compared by means of the four intrinsic quality percentages. In that case the CPT is used as an external point of reference. Such a reference is relevant because the owner of a PPT may not be recognised as a (future) professional colleague, if the PPT is very different from the CPT of the occupational group in which (s)he operates.

Beforehand it is obvious that the four intrinsic qualities may not always be sufficient to express the extent to which a PPT is similar to a CPT. Both can have the same values for concreteness, specificity, complexity and richness and still show substantial differences in content and structure. That is why a method has to be developed to compare the content and structure of a PPT and a CPT. For the validity of such a method it has to take into account that professionals could have developed a discourse typical for their workplace (Bremner, 2012). Such workplace specific jargon could make it hard to determine if certain utterances have the same meaning.

The aforementioned considerations led to the goals of this study: (1) to develop and explore a method for determining open annotated concept maps as representations of CPTs which takes into account the possibility that parts of the CPTs are implicit; (2) to compare the intrinsic qualities of concept maps of CPTs and PPTs; and (3) to develop and explore a method for comparing the content and structure of these maps which reckons with the possibility that elements are expressed in workplace specific jargon. To achieve these goals the conceptual problem has to be addressed first. To this end a conception of CPTs that takes the possibility of differences in opinion between practitioners into account is developed in the theoretical section. 


\section{Conceptual framework}

In this section it is argued that in order to deal with contradicting propositions in the PPTs of practitioners, CPTs could be considered as belonging to a field in the sense of the French sociologist Bourdieu (Hodkinson, Biesta, \& James, 2007). The notion of a field will be conceived as an extension of the notion of a theory as it is defined in the science of logic,

\section{CPTs and contradicting opinions}

A CPT is by definition a theory and in the science of logic a theory is formally defined as a consistent set of propositions, ordered in chains by means of relations of for example a deductive, causal or temporal nature (Gamut, 1991). The required consistency implies that a proposition and its denial cannot both be elements of the same theory. If a CPT is conceived as a theory in this formal sense, the implication is that when two practitioners have opposite views on some professional issue, those views cannot be included in the CPT as two singular statements. Consistency is not violated however when opposed views are inserted by means of a compound statement in the form of a disjunction or an implication. Still, such insertions do not solve the problem of including opposite views in a CPT. In case of disjunctions, the compound statements are examples of the principle of the excluded middle. This principle refers to tautologies that cannot belong to CPTs, as it is impossible to derive any contingent propositions from them (Gamut, 1991). In case of inserting opposite views as the premises of implications - i.e. "If you have as your view that ... then you have to act as ..." - the definition of CPTs used, entails that such implications cannot be elements also because they do not unambiguously prescribe how to act in certain situations. Therefore, the only way to deal with opposite views is to leave them out of 
CPTs, even though the result is CPTs that only portray small parts of the bodies of knowledge, convictions and values relevant in vocational domains.

At first sight, there are two possible approaches to be considered in which this dilemma may be solved. The first approach is to pass off a validated vocational curriculum as a CPT. Such a curriculum seems to be a possibility as it has to contain substantial parts of the relevant knowledge, convictions and values. Moreover, it has to be consistent as students cannot be asked to accept contradicting statements as true. That is why in curricula opposite professional views are often contained as the aforementioned implications. Students learn from these implications that different professional views are possible. However as explained, these implications are one of the reasons that vocational curricula cannot be considered as CPTs. Another reason stems from the observation that the way the content in vocational curricula is structured strongly differs from the ways in which experts structure their professional knowledge (Kondrat, 1992). This may explain why in many vocational institutions there is a debate about the gap between the content of the curriculum and the demands of (regional) occupational groups (Eraut, 2009).

The second approach for solving the dilemma is to pass off the consistent content produced by a knowledge management system (KMS) as a CPT. Such systems are developed to secure the specific knowledge of the employees of a firm or institution as an asset to compete with others (King \& Marks Jr., 2008). The content produced by a KMS describes the firms' practice, so with the same argument used in case of CPTs, the aforementioned implications cannot belong to it. Still, content produced by a KMS cannot be considered as a CPT because the scope of the latter is broader than the scope of the first: a CPT contains all the shared knowledge, convictions and values of an interacting group of practitioners. 
To summarize: when there are substantial opposite views in a vocational domain, it is not feasible to construct a unique CPT of the domain and this problem cannot be solved by conceiving a vocational curriculum or with content produced by a KMS as similar to a CPT.

\section{CPTs considered as elements of a field}

Knowledge management systems have one aspect that could be used to address the problem of opposite views when defining CPTs: its content is produced by a collaborative group of practitioners. When the group of professionals that contribute to a CPT is limited to cooperating practitioners, opposite views may well be placed in different, CPTs. However, as these CPTs all belong to the same domain, there has to be a unifying structure that connects them. The notion of a field introduced by the French sociologist Bourdieu (1996) can be used to unite limited CPTs in the same vocational domain.

In general terms a field can be described as " a configuration of relations between positions objectively defined in their existence and in the determinations they impose upon the occupants, agents or institutions" (Bourdieux as cited in Hodkinson et al., 2008, p. 35). To transform this abstract definition into a notion that can be used in this study we propose to conceive 'positions objectively defined' as CPTs. While working together, practitioners (un)consciously construct a CPT. Such constructs are elements of Popper's third world (Bereiter, 2008): the world of cultural, conceptual artefacts. Just as the 'real' objects in Popper's first world, different people, practitioners, can use, work, explicate and discuss CPTs. That is why they are called objectively defined, in contrast with PPTs which are elements of Popper's second world; the world of mental, subjective objects. Elements of the second world can only be used by the human beings who construct them (Popper, 1972). A CPT represents a 
position in a field because its contributors have a common view on professional issues. In the field of a domain the different professional views are represented in the CPTs that constitute the field.

In this article the term 'field' is used to indicate the complex cohesion of knowledge, values and convictions belonging to a vocational domain. The term 'CPT' is reserved for the knowledge, values and convictions that are actually shared by a specific group of professionals. To describe a field one has to describe the multiple CPTs of which it consists and their mutual relations. These relations could be characterised as influences, because they portray the adaptation of one CPT under influence of another. Influential, outstanding CPTs are called enclaves (Friedman, Sykes, \& Strauch, 2014). They cause a field to change. So fields are dynamic: they evolve in the course of time. Our use of the term 'field' can be characterised as instrumental. It is comparable to the use in physics, where fields were successfully introduced to analyse a class of phenomena whose real nature is not fully understood up until now (Hodkinson et al., 2008).

A premise of the transformation of the abstract definition of Bourdieux is that a group of cooperating professionals actually have a CPT. This assumption is reasonable because professional interaction while cooperating requires a certain homogeneity expressed by a shared body of professional knowledge, convictions and values used when cooperating (Bloor \& Dawson, 1994). It even requires a common discourse to understand each others' point of view (Bremner, 2012). Some differences, e.g. in emphasis, still could exist but are respected by all involved and viewed as supplementing each other, resulting in a collective practice which is presumed being better than the sum of the individual practices (Akkerman \& Bakker, 2011). It is possible that in some cases the premise can be mitigated. For example, in 
vocations where working procedures and conduct are strictly regulated it could be sufficient that practitioners have the same working conditions and types of clients. The relation between CPTs and PPTs

Although CPTs and PPTs belong to different worlds in Popper's universe they do influence each other. On the one hand CPTs continually evolve due to input of the cooperating professionals, the peripheral participation of newcomers and to societal and governmental changes. On the other hand CPTs shape professional habita (Hodkinson et al., 2008) by influencing the language, techniques, division of labour and standards professionals use in their interaction (Alheit, 2009). This implies that CPTs are determined by the PPTs of the relevant occupants, by their practices and by the agents (Sharma, 1997), activity systems (Engeström \& Sannino, 2010) and institutions (e.g. national associations of professionals) in which these occupants participate. The intensity and frequency of the interactions determine which occupants contribute to a CPT. Such interactions can either be local, with occupants engaging with local policy and social conventions, or could extent over different continents as may be expected in a globalising world (Beaverstock, Doel, Hubbard, \& Taylor, 2002).

A CPT does not contain the entire PPTs of the involved professionals. Some of their elements are private by their nature, such as the knowledge of personal strengths, weaknesses and ambitions. Others might not be relevant for the practices in which they interact. On these elements different and even opposite views are possible. It is also possible that the cooperating professionals share tacit knowledge (Endres, Endres, Chowdhurry, \& Alam, 2007). Because such knowledge cannot be made explicit by their owners, it is not contained in their PPTs (Schaap et al., 2009) nor in their socially constructed CPTs. 


\section{Research questions}

As a field could contain a multitude of CPTs, one has to choose a CPT as a point of reference when one wants to compare a PPT with a collective body of professional knowledge, convictions and values of professionals in the domain of the PPT. To explore this further, the goals of this study suggested two empirical research questions: 1) what are the intrinsic qualities of concept maps of CPTs as compared to the PPTs of experts, and 2) to what extent are the content and structure of CPTs and experts' PPTs similar?

\section{Method}

The two research questions were answered by means of a case study in the same domains as in our previous studies: accountancy and teaching English as a foreign language (van den Bogaart et al., submitted). These domains were chosen because they are very different in nature: accountancy is a highly protocollised domain contrary to the teaching domain. To answer the first question an open, annotated group map (Stoyanova \& Kommers, 2002) of a CPT was constructed by a group of five experienced professionals in each of the domains. The construction was supervised by two researches to ensure that the content of the map was shared, formulated in a common language and that workplace jargon was explained. To minimize the possibility that implicit elements of the CPT would be missed, each CPT was commentated by five other experienced practitioners. Their comments were processed in the CPT as long as the construction group agreed. To answer the second research question each CPT was compared with the concept maps of ten (accountancy) or nine (teachers) PPTs of experienced practitioners. To maximize the chance of finding differences of opinion these practitioners did not contribute nor 
comment on the CPT. The choice of the two different domains entailed the extra possibility to compare them. For instance, as a consequence of the highly protocollised character of accountancy contrary to teaching the PPTs of accountants may well be more similar than PPTs of teachers (van den Bogaart, et al., submitted).

\section{Participants}

Experienced accountants and teachers English were selected from the network of a regional university of applied science in the northern part of the Netherlands. 20 Accountants (17 male, 3 female, $M_{\text {age }}=35$ years, $S D=5$ years $)$ and 19 teachers $(5$ male, 14 female, $M_{\text {age }}=44$ years, $S D=10$ years) agreed to participate. 17 Accountants and 18 teachers were alumni of the regional university. The others were educated at other universities in The Netherlands. The participating accountants worked in 15 different small or middle-sized offices with clients in small or middlesized businesses in the region, and had a professional experience of more then five years $(M=16$ years, $S D=6$ years). The teachers worked at 13 different schools and all had experience in the lower years of secondary education with a minimum of more then five years $(\mathrm{M}=17$ years, $S D=9$ years $)$. The accountants who constructed their group map came from five different offices, because it was not possible to find five experienced accountants from one office. This was considered acceptable as they all worked in the same region, in middle-sized offices with local, small and middle-sized firms as clients. Besides this, professional regulations for accountants are very strict and thus similar for each of them. The teachers in the construction group did work in the same school.

\section{Instruments}

Construction of the concept maps of the two CPTs was supported by a module of the dedicated computer program BrainWaever (Van den Bogaart et al., accepted). The 
module allows subjoining examples and clarifying concepts and relations between concepts. All concepts should be connected directly or indirectly with the focus of the concept map: "As a group of accountants/teachers we have professional knowledge of and judgements on ..". The commentary on each group map was also inserted in the concept maps as far as the construction group agreed. Representations of the PPTs of individual professionals were constructed by means of concept maps from BrainWeaver too. In these concept maps the focus was: "As an individual accountant/teacher I have professional knowledge of and judgements on ..". To generate as much information on the PPTs as possible, each map was discussed in a semi-structured interview with its owner. The information derived from the interview was processed in the concept map (van den Bogaart et al., submitted). Trained assessors supported by the assessment module of BrainWeaver (van den Bogaart et al., accepted), determined the percentages that express the intrinsic qualities of the concept maps.

To determine the degree of similarity in content and structure between a concept map of a CPT and a PPT or of two PPTs, a method used in Huyts, de Bruyn and Schaap (2011) was refined. This resulted in a procedure consisting of four steps. The first step is called 'pruning' the two concept maps and makes comparing their content and structure simpler. To prune a concept map one has to look for links between concepts expressing that one of the concepts is a part or an example of the other. That this is the case can be derived from labels such as 'consists of' or 'contains'. If a concept can be conceived as a part or example of another more general concept, the exemplary concept and the link have to be deleted from the map and the exemplary concept has to be inserted as an annotation in the more general concept. The second step consists of forming a list of all the concepts of each of the two 
pruned concept maps. In the third step items from both lists have to be linked by an assessor examining the lists and their corresponding concept maps. A link between an item of a list and an item of the other list expresses the fact that according to the assessor, both items are fully or partly the same. Two concepts can only be deemed as fully the same if their extension and intension are the same (Dummett, 1981). For this purpose, the extension of a concept is defined as the set of all knowledge-elements belonging to the concept (Hampton, 2012). For example the extension of the concept annual account is the set of all propositions describing techniques and regulations regarding the construction of annual accounts. The intension of a concept is defined as the importance and the purpose of the concept in a professional theory. This is operationalized as the order of the concept (primary or sub) in the concept map and as the relations or annotations of the concept expressing its purpose. For instance, a purpose of an annual account could be to provide information for the stock market. Two concepts are to be judged as partly the same if one of the extensions is a part of the other or if the extensions are identical and the intensions are different. For example, the concept profit and loss account is partly the same as the concept annual account because the construction of a profit and loss account is part of the construction of an annual account. By definition an item of a list cannot be fully the same as an item of the other list and at the same time fully or partly the same as another item on that other list. However, an item of a list can be partly the same as several items of the other list.

In the last, and fourth step each item of the two lists is awarded with marks in accordance with table 1 . The degree of similarity in content and structure between two concept maps can then be calculated as the total number of awarded marks divided by the total number of items of both lists, expressed as a percentage. 
Table 1: The award of marks to items

\begin{tabular}{ccc}
\hline Number of links of an item & Interpretation & Mark \\
\hline 0 & No corresponding item & 0 \\
\hline 1 & The item is fully the same as the linked item & 1 \\
\hline$\geq 1$ & The item is partly the same as one or more linked & $1 / 2$ \\
\hline
\end{tabular}

Analysis

For the quantitative analyses of PPTs, five variables were assigned to each concept map. Four variables were defined as the differences between the intrinsic quality percentages of the PPTs and a CPT. The fifth variable expresses the similarity in content and structure between the PPTs and the CPT as a percentage. Low values of the first four variables are an indication that a PPT has the same intrinsic qualities as the CPT. A high value of the fifth percentage indicates that the PPT and the CPT are similar in content an structure. This resulted in: 1) five pairs $H_{0} / H_{1}$-hypotheses in each domain conjecturing that with respect to a variable the CPT and the PPTs are similar/different, and 2) five pairs $H_{0} / H_{l}$-hypotheses, each conjecturing the value of a variable is identical in/differs between both domains.

Due to the small dataset and the large number of variables a Bayesian approach supported by the statistical package $\mathrm{R}$ was used to: 1) calculate the Bayesfactor that expresses the amount of support from the data for $H_{1}$ against $H_{0}$ for each variable in each domain, and 2) determine if there is any support for the conjecture that the two fields differ when characterised by the five variables.

To get more insight in the CPTs, the analysis was supplemented by registration of the handling of differences in opinion, workplace specific jargon and the commentary of colleagues outside the group.

\section{Procedure}


The group construction procedure started with an email sent to the members of the two construction groups explaining the goals and design of the study. In this email they were asked to return a list of topics that, in their view, were important in their profession. To increase the chance that a common language would be used, the two groups each produced a group map of their CPT in a face-to-face session of three hours according to a protocol of three steps. First the lists of topics from the returned emails were discussed in the group to produce a common list of topics every menber agreed upon. The common list was inserted into BrainWeaver and transformed into a collection of concepts, randomly grouped around the focus of the concept map (van den Bogaart et al., in press). In the next step each group member was offered the opportunity to add or delete a concept, to clarify a concept or to explain a relation between two concepts. A discussion on the legitimacy of such an action was not allowed; only an explanation of the proposed action was allowed. If after such an explanation unanimity could be reached, the protocol did allow the action to be executed. Both construction sessions of the two group maps were audio and video taped to obtain information on the negotiation of meaning (Christiansen, 1997).

Each group map was made anonymous and sent for commentary to five experienced colleagues working in other organisations. Their commentary was discussed in the construction groups and again processed in the group maps only if unanimity was reached.

In a period of three months (due to busy time schedules, it was difficult to make appointments) the remaining ten experienced accountants and nine experienced teachers each constructed a concept map of their PPT. First each of them worked a session $(M=52$ minutes, $S D=11$ minutes $)$ with BrainWeaver. A few days later they 
were interviewed on their concept map by telephone $(M=21$ minutes, $S D=4$ minutes).

In each domain two staff members of the regional university of applied science from where the study was conducted and experienced in the assessments of the intrinsic qualities of professional theories (van den Bogaart at al., submitted), determined the intrinsic quality percentages of the nine PPTs and the local CPT. The degrees of similarity in content and structure between the concept maps and the local CPT were assessed by the same staff members also $\left(K_{\text {accountancy }}=.84, N=128\right.$, $\left.K_{\text {teaching }}=.83, N=144\right)$.

\section{Results}

The concept maps produced by the groups of accountants and teachers are shown in Figures 1 and 2. To make a comparison of the two maps easier both are pruned as explained in the method section and annotations to concepts and relations are omitted. All members of both groups appreciated the construction process and stated that the map expressed the main elements of their professional theory.

Group members introduced concepts and relations by means of an explanation with one or two examples. Other members reacted on such a proposal by a reformulation of the concept or relation or by introducing more examples, asking if they understood the proposal well enough. In most cases this led to an insertion of a concept, relation or annotation in the concept map.

The accountancy group disagreed on the insertion of "advice" as a subconcept of the concept products and services at first. In a later stage of the construction process this item was successfully reintroduced as a sub-concept connected to the primary concept product and services by the relation "could lead to". In the teacher group there was discussion on the priority of the concepts language 


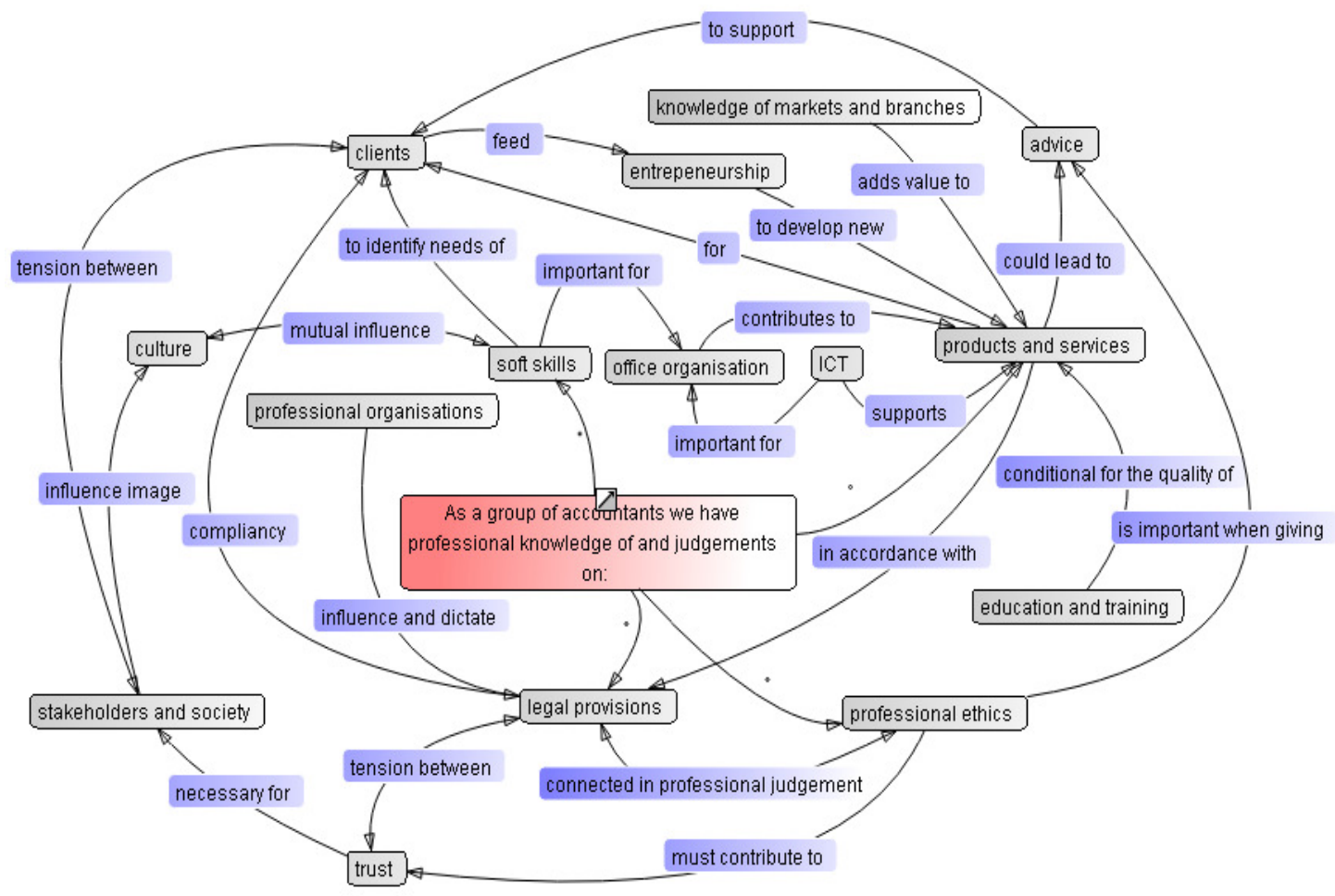

Figure 1. The accountancy group map

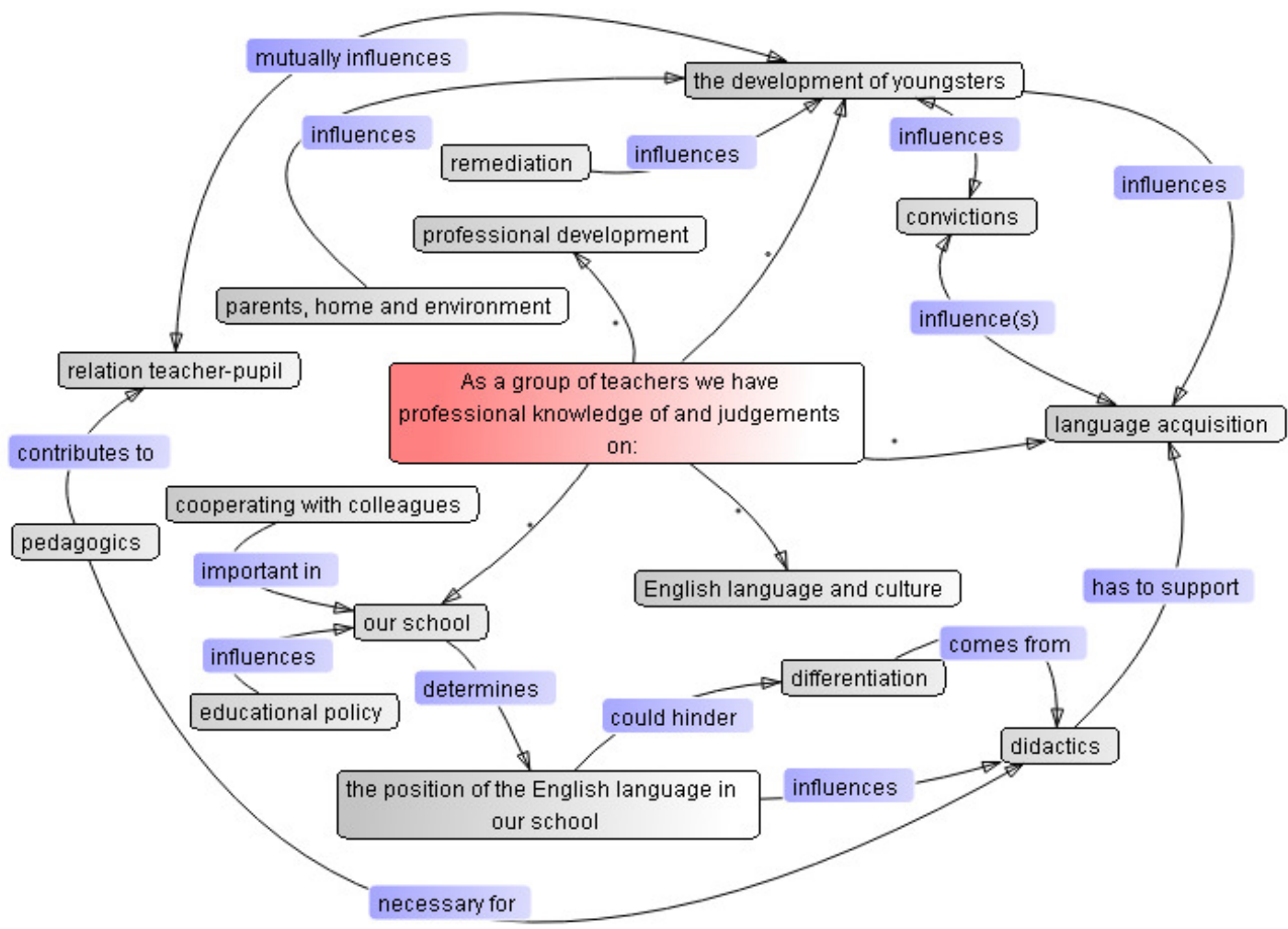


Figure 2. The teaching group map

acquisition versus the development of youngsters in the construction process. In the end both concepts were inserted as primary. The commentary on the group map of the accountants contained one new concept (office organisation) and several explanatory remarks. These were accepted by the construction group and inserted in the concept map. The concept norms and values appeared in two commentaries on the teachers' group map. This concept was not inserted because it immediately led to a discussion about which values and norms that should be. A typical example of professional jargon was found in the teachers' map. As an annotation to the concept didactics the item "N+1" was inserted, which appeared to designate the zone of proximal development according to all group members.

Before pruning, the accountants' group map contained 19 concepts and 28 relations and the teachers' group map 20 concepts and 21 relations. The average numbers of concepts and relations in the concept maps of the PPTs of the ten individual accountants were 15 and 14 . The nine teachers produced an average of also 15 concepts and of 11 relations. The intrinsic qualities of all concept maps are shown in Table 1.

Table 1. The intrinsic qualities of CPTs and PPTs in accountancy and teaching

\begin{tabular}{|c|c|c|c|c|}
\hline & \multicolumn{2}{|c|}{ Accountancy } & \multicolumn{2}{|c|}{ Teaching } \\
\hline & CPT & $M(S D)$ of PPTs & CPT & $M(S D)$ of PPTs \\
\hline Complexity & 87 & $58(15)$ & 73 & $61(8)$ \\
\hline Concreteness & 84 & $80(11)$ & 85 & $88(8)$ \\
\hline Specificity & 68 & $77(12)$ & 83 & $79(9)$ \\
\hline Richness & 75 & $69(17)$ & 50 & 64 (12) \\
\hline
\end{tabular}


As additional information on the intrinsic quality richness the distribution over The distribution over the six domains of knowledge of the annotations to the concepts in the maps is shown in Table 2.

Table 2. The relative frequencies of the domains of knowledge

\begin{tabular}{|c|c|c|c|c|}
\hline & \multicolumn{2}{|c|}{ Accountancy } & \multicolumn{2}{|c|}{ Teaching } \\
\hline & CPT & $M(S D)$ of PPTs & $\mathrm{CPT}$ & $M(S D)$ of PPTs \\
\hline $\begin{array}{c}\text { Vocational field } \\
\text { knowledge }\end{array}$ & 22 & $19(8)$ & 1 & $4(4)$ \\
\hline Organisations & 16 & $5(4)$ & 1 & $5(5)$ \\
\hline $\begin{array}{l}\text { Professional } \\
\text { environment }\end{array}$ & 3 & $16(8)$ & 8 & $7(5)$ \\
\hline Target group & 7 & $14(6)$ & 34 & $19(7)$ \\
\hline $\begin{array}{l}\text { Technical- } \\
\text { instrumental }\end{array}$ & 41 & $37(15)$ & 49 & $45(13)$ \\
\hline $\begin{array}{c}\text { Self-knowledge and } \\
\text { development }\end{array}$ & 12 & $9(7)$ & 6 & $19(8)$ \\
\hline
\end{tabular}

The average similarity of content and structure between the concept maps of the CPT and the PPTs is $56 \%(S D=11)$ in accountancy, and $40 \%(S D=6)$ in teaching. An outstanding difference between the CPT and PPTs in accountancy is the focus. According to the concept map the CPT covers both audit and non-audit services, while PPTs usually focus only on one of these aspects depending on the practice of its owner. The CPT of the teachers also has a broader focus then the individual maps. This is expressed by the fact that the concept map of the CPT has five primary concepts covering different aspects of the profession, while in the PPTs the focus is narrower: pedagogical, stressing knowledge transmission or classroom management, etc.

In Table 3 the Bayesian factors ( $B f s$ ) are displayed together with the $H_{1^{-}}$ hypotheses belonging to it, for which there is positive support $(B f \geq 3$ ), strong 
support $(B f \geq 12)$ or very strong support $(B f>150)$ (Jackman, 2009). For all other $H_{l}$ there is no support $(B f<3)$ and therefore these are omitted.

Table 3. Bayesian factors and $H_{1}$-hypotheses

\begin{tabular}{|c|c|}
\hline$H_{1}$-hypothesis & $B f$ \\
\hline The complexity of the accountants' PPTs differs from the CPT & 134,8 \\
\hline The complexity of the teachers' PPTs differs from the CPT & 13,9 \\
\hline The richness of the teachers' PPTs differs from the CPT & 5,1 \\
\hline The content and structure of the accountants' PPT differs from the CPT & 23000 \\
\hline The content and structure of the teachers' PPT differs from the CPT & 27000 \\
\hline $\begin{array}{l}\text { Both domains differ in the degree of resemblance in content and structure } \\
\text { between CPT and PPTs. }\end{array}$ & 25,0 \\
\hline
\end{tabular}

\section{Conclusion and Discussion}

Regarding the first research question the $B f$ shows that the complexity of the accountancy CPT differs from the PPTs (87 vv. 58). In the educational domain the difference is smaller (73 vv. 61) but also strongly supported by the data. The other intrinsic qualities do not lead to significant differences between CPTs and PPTs except the quality richness in the educational domain (64 vv. 50). Differences in complexity can be explained from the observation that both CPTs cover more aspects of the profession than the PPTs do. In case of accountancy this could be a result of the fact that the members originated from different firms with similar, but different practices. The teachers who constructed the group map work in the same school. Therefore, it is plausible that they have developed a CPT, which incorporates most of their PPTs (Bryk, 1988). The difference in richness between the teachers' CPT and the PPTs can be explained by the data in Table 2. In the PPTs there is one prominent domain more then in the CPTs: self-knowledge and development. To know ones 
weaknesses and strengths is important for teachers (Hamachek, 1999). However, such personal knowledge cannot be part of a CPT.

Comparing the four intrinsic qualities of the PPTs in the two domains did not result in significant differences. This is in accordance with the results stated in van den Bogaart et al. (submitted). The biggest difference between the CPTs is the quality richness ( 75 vv. 50). The explanation can again be found in Table 2: the six domains of knowledge of the accountants' CPT are distributed more evenly than in the teachers' CPT. Notably knowledge of the vocational field and of organisations (in general) are almost absent in the CPT of the teachers. Whether this is a more common phenomenon of CPTs in education is a question for further research.

Regarding the second research question, the data provide very strong support in both domains for the hypothesis that CPTs and PPTs differ in content and structure. This is not surprising, because individual shortcomings and even blind spots may be compensated by a collective. There is strong support for the hypothesis that the difference in content and structure between CPT and PPTs is larger in education than in accountancy. This may indicate that the structure of the field of accountancy differs from the educational field in terms of "density": professional differences between individual (groups of) experts might on average probably be larger in education than in accountancy. This is in accordance with the earlier provided characterisation of the accountancy field as highly protocollised contrary to the less structured educational field.

The two case studies probably reveal just fragments of the two fields. Although indications of professional disputes were found, the controversy on non-audit services in accountancy and on the prominence of the pedagogical dimension in education, it is not clear to which degree these controversies could lead to contradicting 
propositions that cannot be united in a CPT. In fact, in case of the two produced CPTs both groups found ways to capture opposite views in the group maps. The accountancy group used the relation "could lead to" to connect the non-audit service "advice" to audit services and the teacher group declared both the pedagogical and the instructional dimension as primary. It is not clear whether both choices lead to CPTs that function in practice and whether there are CPTs in both fields that represent a more distinct point of view. These are interesting questions for further research too.

Finally it is interesting to study how PPTs, CPTs and the surrounding field influence each other. This is important for vocational education and professional development as awareness of CPTs and the surrounding field in relation to one's own PPT helps to find one's place in a domain. 


\section{References}

Akkerman, S. F., \& Bakker A. (2011). Learning at the boundary: An introduction. International Journal of Educational Research. 50, 1-5.

Alheit, P (2009). Biographical Learning within the new lifelong learning discourse. In Illeris K. (Ed.), Contempory theories of learning (pp. 116-128). London: Routledge.

Anastasi, A., Urbina, S. (1997). Psychological Testing (Seventh ed.). Upper Saddle River, NJ: Prentice Hall.

Ashbaugh, H., LaFond, R., \& Mayhew, B. W. (2003). Do non-audit services compromise auditor independence? Further evidence. The Accounting Review. 78(3), 611-639.

Bereiter, C. (2002). Education and Mind in the Knowledge Age. Mahwah (NJ): Lawrence Erlbaum Associates, Publishers.

Bloor, G. \& Dawson, G. (1994). Understanding professional culture in organisational context. Organisation Studies, 15(2), 275-296.

Bremner, S. (2012). Socialisation and the acquisition of professional discourse: a case study in the PR industry. Written Communication, 29(1), 7-32.

Bryk, A. S., \& Driscoll, M. E. (1988). The High School as Community: Contextual Influences and Consequences for Students and Teachers.

Bourdieux, P. (1996). The rules of art. Cambridge: Polity.

Christiansen, I. M. (1997). When negotiation of meaning is also negotiation of task: analysis of communication in an applied mathematics high school course. Educational Studies in Mathematics, 34(1), 1-25.

de Bruijn, E., \& Nieuwenhuis, L. F. M. (1994). Education between profession and market: the development of professional training. In W.J. Nijhof \& J. N. 
Streumer (Eds.), Flexibility in training and vocational education (pp. 109-131). Utrecht: Lemma.

De Long, D. W., \& Fahey, L. (2000). Diagnosing Cultural barriers to knowledge management. Academy of Management Executive. 14 (4), 113-126.

Dummett, M. (1981). Frege, philosophy of language. London: Duckworth.

Endres, M. L., Endres, S. P., Chowdhury, S. K., \& Alam I. (2007). Tacit knowledge sharing, self-efficacy theory, and application to the open source community. Journal of Knowledge Management, 11(3), 92-103.

Engeström, Y, \& Sannino, A. (2010). Studies of expansive learning: Foundations, findings and future challenges. Educational Research Review, 5(1), 1-24.

Eraut, M. (2009). Transfer of knowledge between education and workplace settings. In H. Daniels, H. Lauder, \& J. Porter (Eds.), Knowledge, values and educational policy; a critical perspective (pp. 1-17). New York, NY: Routledge.

Friedman, V. J., Sykes, I. \& Strauch, M. (2014). Expanding the realm of the possible: enclaves and the transformation of fields. Academy of Management Proceedings 2014(1), 1-30.

Gamut, L. T. F. (1991). Logic, language, and meaning. Chicago, IL: The University of Chicago Press.

Hamachek, D. (1999). Effective teachers: what they do, how they do it, and the importance of self-knowledge. In R. P. Lipka, \& T. M. Brinthaupt (Eds.). The role of self in teacher development (pp. 189-224). Albany, NY: State University of New York Press.

Hampton, J. A. (2012). Thinking intuitively: the rich (and at times illogical) world of concepts. Current Directions in Psychological Science, 21(6), 398-402. 
Hodkinson, P., Biesta, G., \& James, D. (2008) Understanding Learning Culturally:

Overcoming the dualism Between Social and Individual Views of Learning. Vocations and Learning, 1, 27-47.

Huyts, P. M., de Bruijn, E., \& Schaap, H. (2011). Revealing Personal professional Theories. Quality and Quantity, 45, 783-800.

Jackman, S. (2009). Bayesian analysis for the social sciences. Chichester: John Wiley \& Sons, Ltd.

King, W. R. \& Marks Jr., P. V. (2008) Motivating knowledge sharing through a knowledge management system. Omega, 36, 131-146.

Kondrat, M. E. (1992). Reclaiming the Practical: Formal and Substantive Rationality in Social Work Practice. Social Service Review, 66(2), 237-255.

Popper, K. R. (1972). Objective knowledge; an evolutionary approach. Oxford, At the Clarendon Press.

Proctor, H., Brownlee, P., \& Freebody, P. (Eds.) (2015). Controversies in education: orthodoxy and heresy in policy and practice. Cham: Springer.

Schaap, H., de Bruijn, E., van der Schaaf, M. F., Baartman, L. K. J., \& Kirschner, P. A. (2011). Explicating students' personal professional theories in competence based vocational education through multi-method triangulation. Scandinavion Journal of Educational Research, 55, 567-586.

Schaap, H., de Bruijn, E., van der Schaaf, M. F., \& Kirschner, P. A. (2009). Students' personal professional theories in competence-based vocational education: the construction of personal knowledge through internalisation and socialisation. Journal of Vocational Education and Training, 61, 481-494.

Sharma, A. (1997). Professional as agent: knowledge asymmetry in agency exchange. Academy of Management Review, 22(3), 758-796. 
Stoyanova, N. \& Kommers, P. (2202) Concept mapping as medium of shared cognition in computer-supported collaborative problem solving. Journal of Interactive Learning Research, 13(1-2), 111-133.

van den Bogaart, A. C. M., Bilderbeek, R., Schaap, H., Hummel H. M. F., \& Kirschner, P. A. A. (2015). Computer Supported Method to Reveal and Assess Personal Professional Theories in Vocational Education. Manuscript accepted for publication in Technology, Pedagogy and education.

van den Bogaart, A. C. M., Schaap, H., Hummel H. M. F., \& Kirschner, P. A. Combining Concept Maps and Interviews to Produce Representations of Personal Professional Theories: Effects of Order and Vocational Domain. Manuscript submitted for publication.

Wenger, E., McDermott, R., \& Snyder, W. (2002). Cultivating communities of practice: A guide to managing knowledge. Boston (M): Harvard Business school Press.

Wilson, S. M., \& Berne, J. (1999) Teacher learning and the acquisition of professional knowledge: an examination of contemporary professional development. Review of Research in Education, 24, 173-209. 